\title{
Covid-19 Diagnosis by Artificial Intelligence Based on Vibraimage Measurement of Behavioral Parameters
}

\author{
Viktor Minkin ${ }^{1}$, Alexander Bobrov², Valery Akimov', Eugeniia Lobanova1, Yana Nikolaenko1, \\ Oleg Martynov ${ }^{1}$, George Zazulin ${ }^{1}$ \\ ${ }^{1}$ Elsys Corp., St. Petersburg, Russia \\ ${ }^{2}$ State Research Center-Burnasyan Federal Medical Biophysical Center of Federal Medical and Biological Agency (SRC-FMBC) of \\ Russia, Moscow, Russia \\ Email: ^minkin@elsys.ru, baf-vcmk@mail.ru
}

How to cite this paper: Minkin, V., Bobrov, A., Akimov, V., Lobanova, E., Nikolaenko, Y., Martynov, O. and Zazulin, G. (2020) Covid-19 Diagnosis by Artificial Intelligence Based on Vibraimage Measurement of Behavioral Parameters. Journal of Behavioral and Brain Science, 10, 590-603. https://doi.org/10.4236/jbbs.2020.1012037

Received: November 26, 2020

Accepted: December 28, 2020

Published: December 31, 2020

Copyright $\odot 2020$ by author(s) and Scientific Research Publishing Inc. This work is licensed under the Creative Commons Attribution International License (CC BY 4.0).

http://creativecommons.org/licenses/by/4.0/

\begin{abstract}
The hypothesis of behavioral parameters dependence measured from person's head movements in quasi-stationary state on COVID-19 disease is discussed. Method for determining the dependence of vestibular-emotional reflex parameters on COVID-19, various diseases and pathologies are proposed. Micro-movements of a head for representatives of the control group (with a confirmed absence of COVID-19 disease) and a group of patients with a confirmed diagnosis of COVID-19 were studied using vibraimage technology. Parameters and criteria for the diagnosis of COVID-19 for training artificial intelligence (AI) on the control group and the patient group are proposed. 3-layer (one hidden layer) feedforward neural network $(40+20+1$ sigmoid neurons) was developed for AI training. AI was firstly trained on the primary sample of patients and a control group. Study of a random sample of people with trained AI was carried out and the possibility of detecting COVID-19 using the proposed method was proved a week before the onset of clinical symptoms of the disease. Number of COVID-19 diagnostic parameters was increased to 26 and AI was trained on a sample of 536 measurements, 268 patient measurement results and 268 measurement results in the control group. The achieved diagnostic accuracy was more than 99\%, 4 errors per 536 measurements ( 2 false positive and 2 false negative), specificity $99.25 \%$ and sensitivity $99.25 \%$. The issues of improving the accuracy and reliability of the proposed method for diagnosing COVID-19 are discussed. Further ways to improve the characteristics and applicability of the proposed method of diagnosis and self-diagnosis of COVID-19 are outlined.
\end{abstract}




\section{Keywords}

Vibraimage, Health, Behavior, Artificial Neural Networks, ANN, Artificial

Intelligence, AI, Vestibular-Emotional Reflex, Diagnosis of Diseases,

Telemedicine, COVID-19

\section{Introduction}

Currently, the COVID-19 epidemic is only growing and at the time of this writing, record numbers of infected people were recorded in the world [1] [2]. One of the significant factors in the spread of the pandemic is the significant time between a patient's infection and the onset of symptoms of COVID-19 disease [3]. There are a number of biochemical diagnostic methods for COVID-19, which can mainly be divided into blood tests (antibody) and taking nasopharyngeal swabs (PCR) [4] [5] [6], however, in real conditions most countries have significant time between taking a test and receiving COVID-19 diagnostic result [7]. For example, in Russia this time can be more than 2 weeks due to many different reasons, despite the recommendation of obtaining test results within 48 hours [8]. At the same time, even the obligatory 48 hours is an unbearably long time for the testing procedure, for example, at the airports or during the pre-shift control of workers on factories, during attending public events, when children go to school, etc. Thus, it turns out that known biochemical methods for diagnoses diseases with a long incubation period, in principle, cannot be a barrier to the spread of infections [9]. This is confirmed by the current spread of COVID-19 in the world, despite all the efforts made by the WHO and various countries. The use of temperature control as a diagnosis of COVID-19 in crowded places creates only the illusion of safety, since the asymptomatic and temperature normal carriers of COVID-19 poses the greatest danger to infecting others [10].

At the same time, there are a number of alternative technologies for detecting COVID-19 that are not directly based on biochemical analysis of the virus, but analyze the indirect consequences of its impact on the body. One such known method is the specific odor of the disease, which can be detected by specially trained dogs [11]. It is clear that such a method for detecting a disease cannot become a mass protection of the population, but it shows the fundamental possibility of diagnosing COVID-19 according to the consequences of the effect of the virus on a human body.

Another alternative way to diagnose COVID-19 is diagnostics by the sound of a cough, performed by trained artificial intelligence [9]. According to the authors, cough is a significant indicator of the disease, the cough of a healthy person and a person with COVID-19 varies significantly in parameters, and artificial intelligence (AI) trained on control group and patients group finds significant differences in cough parameters. At the same time, the proposed diagnostic method using AI in terms of cough parameters has obvious practical limitations, since after coughing each person, it is necessary to sanitize the premises. In ad- 
dition, not all COVID-19 infected patients [12] have lung involvement, and such patients should not be identified by cough parameters. Moreover, medical recommendations can hardly contain a requirement to cough, since this method can lead to coughing fits in a certain number of patients under studyю

At the beginning of the COVID-19 epidemic was proposed method for diagnosing diseases by the micromovements analyzing of a human head using vibraimage technology [13] [14] [15]. Was supposed to use the analysis of the synchronization of physiological processes as one of the main indicator $\Sigma[\mathrm{R}]$ of human health, with the second indicator $\Sigma[\Delta \mathrm{M}]$ reflecting the correspondence of reflex movements of the human head to the obtained head movement template for a relatively large number $(15,000)$ of healthy people [16]. The possibility of vibraimage technology using to identify a specific disease previously was confirmed by express diagnosis of prostate cancer, when it was proved that vestibular-emotional reflex is sensitive to oncology changes in the body [17]. Violation of the normal rhythm of reflex micromovements of the head can occur both in the case of mental changes and in the case of physiological pathology [18]. However, our studies in the first half of 2020 showed that integral psychophysiological parameters determined by micromovements of the head and used to determine the level of functional health or oncology of the prostate turned out to be weakly sensitive to identifying asymptomatic carriers of COVID-19, namely, they pose the main danger in the spread of infection. Considering that $\mathrm{AI}$ showed quite well in solving precisely exact problems [19], where is possible to conduct a study by independent methods and form a control group and a group of patients using standard methods of biochemical and radiological diagnostics. Therefore, the developers of vibraimage technology created an artificial neural network and train AI according to the vibraimage parameters of the indicated groups.

\section{Materials and Method}

\subsection{Pretesting Materials and Method}

144 measurements of the micromovements of the head of patients parameters with a confirmed diagnosis of COVID-19 (136 patients with confirmation of Covid-19 by CT and PCR in the active phase and 8 patients with asymptomatic COVID-19) formed the group of patients. All measurements were done by VibraHT program [20] or HealthTest [21] program. VibraHT is professional program giving all setting and parameters information to users. VibraHT is more detailed, includes more parameters for assessment than HealthTest. HealthTest is simplified program version operating in default settings and gives only main health parameters to users. Both programs VibraHT and HealthTest have identical processing algorithm for COVID-19 diagnosis. The measurements were done between May and June 2020. The age of the patients was from 25 to 75 years, the male-to-female ratio 60 - 40\%. Ethnic composition-100\% Russian. The demographic parameters of the control group were matched identically to 
the patient group. To form control group, 144 measurements of head micromovements parameters of people with a complete absence of COVID-19 symptoms and a negative PCR test for COVID-19 were used. Web cams MS LifeCam Cinema and MS LifeCam Studio captured video information of person head. Cameras resolution was set $640 \times 480$, frame frequency $30 \mathrm{f} / \mathrm{s}$, b/w mode on in settings. Person position was about $50 \mathrm{~cm}$ from webcam, head size in horizontal line was more than 200 pixels. Image Quality test level by VibraHT and HealthTest programs was higher than $80 \%$ for all accepted testing results.

The relatively small number of measurements in the patient group and the control group was determined by two factors. The presence of lockdown introduced in the country (Russia) for the period of obtaining data and the legal uncertainty of biometric data capturing, which include the results of micromovements measurements of a human head. All participants in the study of human head micromovements using vibraimage technology, the results of which are used in this work, signed an Agreement on anonymized using of biometric data when publishing research results.

To process measured results in Elsys Corp, St. Petersburg, Russia, artificial neural network (ANN) was developed and trained with simple three-layer feedforward structure, shown in Figure 1.

The values of micromovements parameters of a human head T1-T10 [16] were used as input parameters on the first network layer in the following order for each input sigmoid neuron of the first layer of the neural network:

$M(\mathrm{~T} 1), \mathrm{S}(\mathrm{T} 1), \mathrm{M}(\mathrm{T} 2), \mathrm{S}(\mathrm{T} 2), \mathrm{M}(\mathrm{T} 3), \mathrm{S}(\mathrm{T} 3), \mathrm{M}(\mathrm{T} 4), \mathrm{S}(\mathrm{T} 4), \mathrm{M}(\mathrm{T} 5), \mathrm{S}(\mathrm{T} 5)$, M(T6), S(T6), M(T7), S(T7), M(T8), S(T8), M(T9), S(T9), M(T10), S(T10).

In vibraimage technology $\mathrm{T} 1-\mathrm{T} 10$ parameters are determined in the way to have minimum correlation with each other [13] [16], therefore a limited number of parameters characterizes reflex micromovements of a head as informative as possible [18].

Where: $\mathrm{M}(\mathrm{Ti})$ - median value of $\mathrm{i}$ parameter during the measurement.

$\mathrm{S}$ (Ti)-SD of i parameter during the measurement.

All input parameters $\mathrm{M}(\mathrm{Ti})$ and $\mathrm{S}(\mathrm{Ti})$ are scaled to the range $[0 \ldots 1]$.

After the formation of the neural network with the configuration shown in Figure 1, the AI was trained on the control and patient databases groups. The training was carried out on Sony Vaio computer with i7 processor, RAM $=16 \mathrm{~Gb}$ and took 24 hours.

After training, the AI discriminated two groups with an accuracy of $94 \%$, out of 288 results there were 15 errors, 7 false results in the patient group and 8 false results in the healthy group. Of the 7 false results in the patient group, 4 were related to the measurements of patients at the late stage of COVID-19 but were left in the training sample, since the ability to infect at a late stage of the disease has not yet been sufficiently studied [22]. The AI discrimination function was tuned in such a way that when the number of measurements in the training samples is equal, the threshold value for accepting solution to place the result as negative or positive is 0.5, which is reflected in Figure 2. 


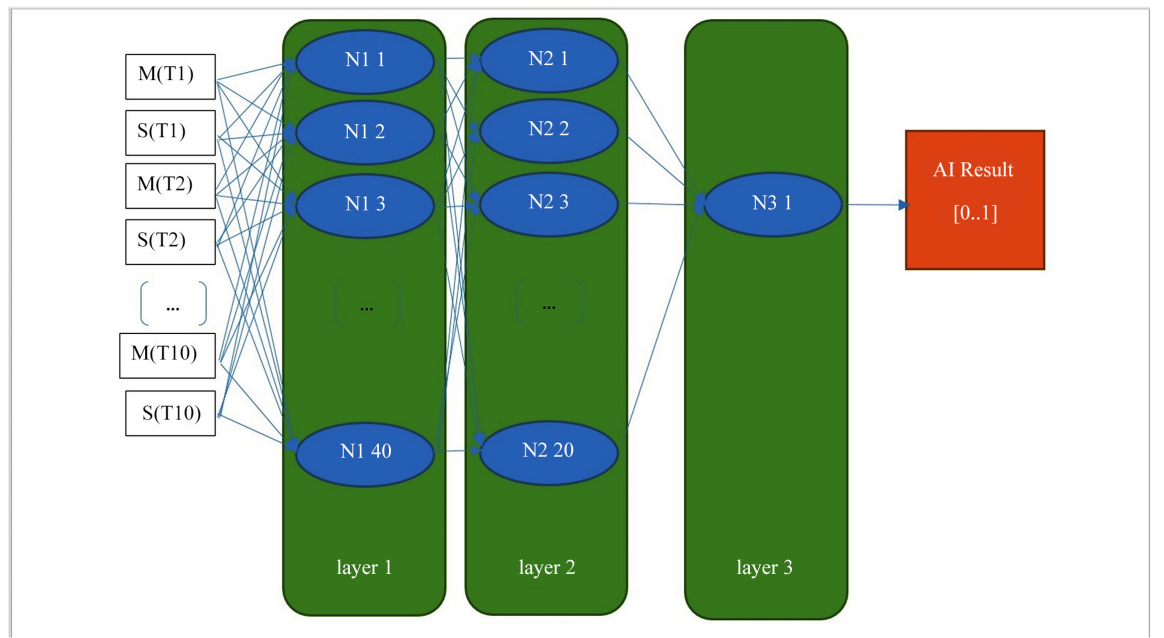

Figure 1. Block diagram structure of feedforward neural network for COVID-19 diagnosis.

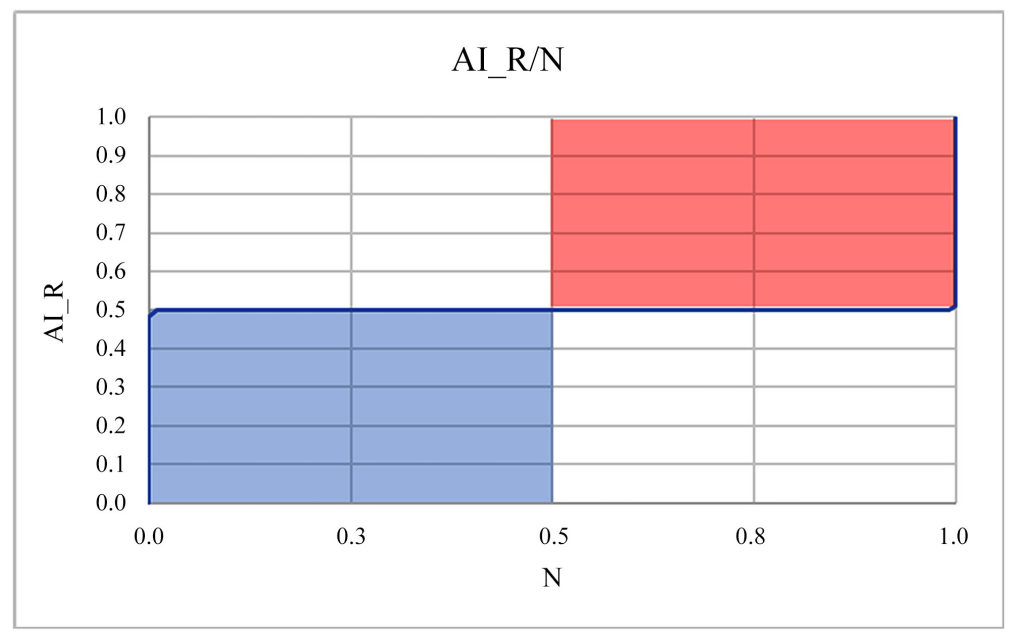

Figure 2. AI decision function for groups discrimination.

Following AI discrimination function was set when the number of measurements in the training groups is equal, the threshold value for accepting solution to place the result as negative or positive is 0.5 , shown on Figure 2 .

AI decision results with a score of less than 0.5 are considered as negative COVID-19, and results greater than 0.5 are considered as positive COVID-19.

\subsection{Pretesting AI for COVID-19 Diagnosis}

Based on the results obtained in the course of pretest studies, changes were made to HealthTest and VibraHT programs and, in addition to the integral health indicators $\Sigma[\mathrm{R}]$ and $\Sigma[\Delta \mathrm{M}]$, was added the COVID-19 probability indicator, calculated by $\mathrm{AI}$ on discrimination input data.

Since the Federal law of experimental legal regimes in the sphere of digital innovations in the Russian Federation [23] appeared at the beginning of August 2020, which allows the use of telemedicine in the practical area, Elsys Corp, St. Petersburg, Russia, decided to monitor each employee's pre-shift checking by 
the updated HealthTest program from September 1, 2020.

On October 20, by pre-shift control, one of Elsys's employees (male, 66 years old) showed $100 \%$ probable of COVID-19. The result of that measurement by VibraHT program is shown in Table 1.

The health indicators shown in Table 1 and the norms for them are described in the HealthTest and VibraHT programs Manuals [20]. Note that not only the employee's integral health indicators at the time of October 20 were normal, but all other behavioral and psycho-emotional parameters measured by the VibraHT program [20] and shown in Figure 3 were also within the normal range.

Naturally, the employee suspected of COVID-19 was quarantined, although his temperature at that moment was 36.5 and he did not have any external signs of SARS or COVID-19. The first signs of COVID-19 (temperature $37.5^{\circ} \mathrm{C}$ headache, weakness) appeared on Monday 26 October, i.e. 6 days after the detection of COVID-19 by the HealthTest program. On October 27, a doctor was called who prescribed treatment (Azithromycin $500 \mathrm{mg} .1$ tab. 9 days +3 days, Ingavirin $90 \mathrm{mg} .1$ tab. 1 time per day, 7 days, Thrombo ACC $75 \mathrm{mg}$. gpo 1 tab. 1 time per day, 10 days, Grippferon (nasal drops) 5 times a day, 7 days) and gave a referral to CT, which revealed $25 \%$ lung damage.

Selected data from the monitoring of integral health indicators determined by the HealthTest program [21] for the given patient in the course of illness and treatment shown in Figure 4.

The given data from Figure 4 show the main trends in changes of both integral health indicators and the dynamics of COVID-19 diagnostics. Green color in Health of Figure 4 cell shows position of health indicators inside norms. Yellow color in Health table cell shows outside position for one indicator out of norms. Green color shows normal Health based on two health indicators. AI indicator from Figure 4 show the result of COVID-19 diagnosis by HealthTest program, also green is normal, red is COVID. Taken on the same date of last measurement 16 November 2020 enzyme immunoassay analysis indicates IgA $=4.33$ and $\operatorname{IgG}=3.46$ and having antibodies show immunity to COVID-19.

The dynamics of health indicators [15] shows a noticeable deterioration in general health indicators in the active phase of COVID-19 and almost their recovery after about 3 weeks from the moment of illness for this particular patient. Health indication comes from green zone before disease to red zone in active COVID-19 phase to green zone after COVID-19 treatment on Figure 4.

Table 1. Detection of COVID-19 with normal integral health indicators by HealthTest program.

\begin{tabular}{cc}
\hline 10-20-13-16 & Indicator (norms) \\
\hline Health & $\Sigma[\mathrm{R}]=29.56(>20.0)$ \\
$\mathrm{t}=61 \mathrm{~s}$ & $\Sigma[\Delta \mathrm{M}]=3.92(<4.0)$ \\
& $\mathrm{AI}=100.00(<50)$ \\
\hline
\end{tabular}




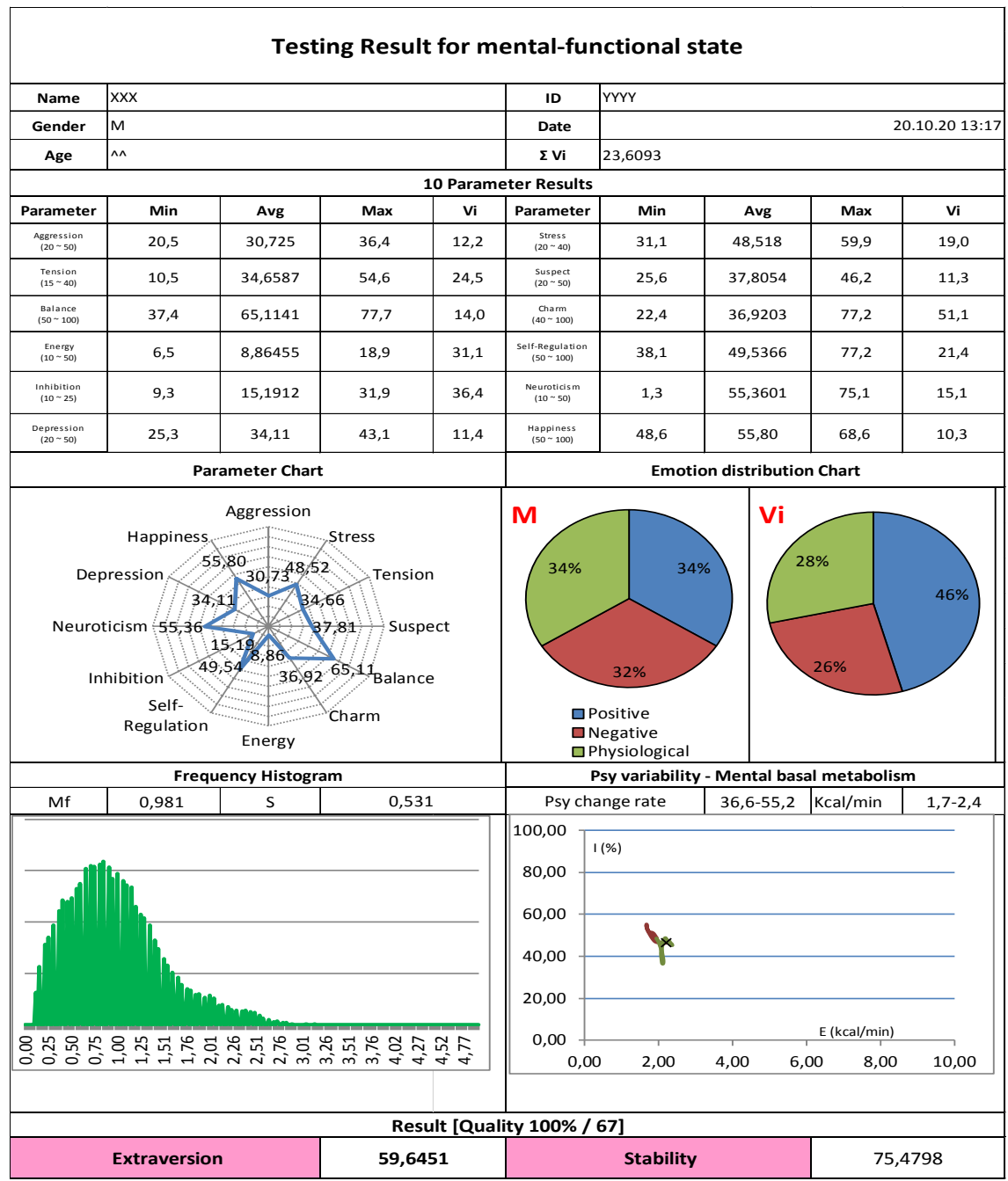

Figure 3. Psychophysiological parameters values for early detection of COVID-19 by VibraHT program main menu.

\begin{tabular}{|c|c|c|c|}
\hline Data & $\begin{array}{c}\text { Health Indicator } \\
\text { (norms) }\end{array}$ & Data & $\begin{array}{c}\text { Health Indicator } \\
\text { (norms) }\end{array}$ \\
\hline 10-19-11-03 & & $10-31-19-55$ & \\
\hline Health & $\Sigma[R]=20,13(>20,0)$ & Health & $\Sigma[\mathrm{R}]=18,82(>20,0)$ \\
\hline \multirow[t]{2}{*}{$t=61 s$} & $\Sigma[\Delta \mathrm{M}]=1,56(<4,0)$ & $\mathrm{t}=181 \mathrm{~s}$ & $\Sigma[\Delta \mathrm{M}]=3,27(<4,0)$ \\
\hline & $\mathrm{Al}=0,00(<50)$ & & $\mathrm{AI}=100,00(<50)$ \\
\hline $10-20-13-16$ & & $11-01-14-13$ & \\
\hline Health & $\Sigma[\mathrm{R}]=29,56(>20,0)$ & Health & $\Sigma[\mathrm{R}]=13,63(>20,0)$ \\
\hline \multirow[t]{2}{*}{$\mathrm{t}=61 \mathrm{~s}$} & $\Sigma[\Delta \mathrm{M}]=3,92(<4,0)$ & $\mathrm{t}=180 \mathrm{~s}$ & $\Sigma[\Delta \mathrm{M}]=4,23(<4,0)$ \\
\hline & $\mathrm{AI}=100,00(<50)$ & & $\mathrm{AI}=99,92(<50)$ \\
\hline $10-25-09-47$ & & $11-10-12-31$ & \\
\hline Health & $\Sigma[\mathrm{R}]=21,90(>20,0)$ & Health & $\Sigma[\mathrm{R}]=22,26(>20,0)$ \\
\hline \multirow[t]{2}{*}{$\mathrm{t}=180 \mathrm{~s}$} & $\Sigma[\Delta \mathrm{M}]=3,29(<4,0)$ & $\mathrm{t}=180 \mathrm{~s}$ & $\Sigma[\Delta \mathrm{M}]=2,27(<4,0)$ \\
\hline & $\mathrm{AI}=100,00(<50)$ & & $\mathrm{AI}=95,73(<50)$ \\
\hline $10-27-18-09$ & & $11-16-11-56$ & \\
\hline Health & $\Sigma[\mathrm{R}]=19,99(>20,0)$ & Health & $\Sigma[\mathrm{R}]=30,26(>20,0)$ \\
\hline \multirow[t]{2}{*}{$\mathrm{t}=180 \mathrm{~s}$} & $\Sigma[\Delta \mathrm{M}]=4,05(<4,0)$ & $\mathrm{t}=180 \mathrm{~s}$ & $\Sigma[\Delta \mathrm{M}]=2,74(<4,0)$ \\
\hline & $\mathrm{AI}=0,00(<50)$ & & $\mathrm{AI}=0,00(<50)$ \\
\hline \multicolumn{4}{|l|}{$10-28-17-57$} \\
\hline Health & $\Sigma[\mathrm{R}]=15,14(>20,0)$ & & \\
\hline \multirow[t]{2}{*}{$\mathrm{t}=180 \mathrm{~s}$} & $\Sigma[\Delta \mathrm{M}]=4,44(<4,0)$ & & \\
\hline & $\mathrm{AI}=0,00(<50)$ & & \\
\hline
\end{tabular}

Figure 4. Detection of COVID-19 and integral health indicators dynamics by HealthTest Program in case of proven COVID-19 disease. 


\section{Analysis of Results}

\section{Materials}

COVID-19 detection of our employee, and then his wife COVID-19 infection, allowed us significantly (+102 measurements) replenish the database of measurements of patients with confirmed COVID-19 and naturally collect more data for the control group.

At the time of writing this article, the number of patient's measurements with confirmed COVID-19 in patient group was 268 measurements and 268 measurements in the control group (536 measurements in total). The structure of the neural network, shown in Figure 1, was left the same and the number of input vibraimage parameters, characterizing the micromovements of a head, was increased from 20 to 26.

In the course of the main study, the AI of the neural network (with the configuration shown in Figure 1) was trained on the augmented measurement databases of the control group and the patient group. The training was carried out on the same Sony Vaio computer with i7 processor, RAM = $16 \mathrm{~Gb}$ and took 1 hour. After training AI discriminated two groups of 268 measurements with an accuracy higher than 99\%, out of 536 results, 4 errors were detected, 2 false positive results in the group of patients and 2 false negative results in the group of healthy people. Figure 5 shows the sensitivity-specificity curve for the obtained sample of 536 results in accordance with GOST R 53022.3 in 2008.

Resulting sensitivity value was $99.25 \%$, the resulting specificity value was the same $99.25 \%$.

The distribution density of the sensitivity and specificity values for the negative and positive groups to COVID-19 for the sample of 536, depending on the presented threshold values of the AI decision results, shown in Table 2 based on Excel calculation of AI data.

Data shown on Table 2 indicates a slight difference between the results obtained from the ideal broken sorting of patient groups and the control. Differences in sensitivity values of real values did not exceed the deviation in the fourth decimal place for both groups, and the number of non-ideal measurements was minimal. The total error of sorting by groups (4 results out of 536) was less than one percent, respectively, the overall accuracy of sorting by groups was $99.25 \%$.

Figure 6 shows the coefficients of significance for 28 input vibraimage parameters when processing the first line of the neural network, showing the different influence of the input parameters on the sorting result. The yellow line marks the maximum significance coefficients obtained for each input parameter.

Most likely, input parameters with insignificant data in the highlighted row of Figure 6 (for example, significance less than 10 can later be replaced with other input parameters that characterize more informative parameters of micromove- 
ments of the human head and provide a higher quality of group classification in the diagnosis of COVID-19. Pay attention that highest significant level has SD of the following behavior parameters-aggression, anxiety, energy, neuroticism, happiness $(\mathrm{S}(\mathrm{T} 1)=44 ; \mathrm{S}(\mathrm{T} 3)=37 ; \mathrm{S}(\mathrm{T} 7)=36 ; \mathrm{S}(\mathrm{T} 10)=38 ; \mathrm{S}(\mathrm{T} 12)=37)$, showing the main difference in behavioral parameters for patient and control groups. All current statistics based on AI solution and is difficult to calculate or confirm by other statistics methods.

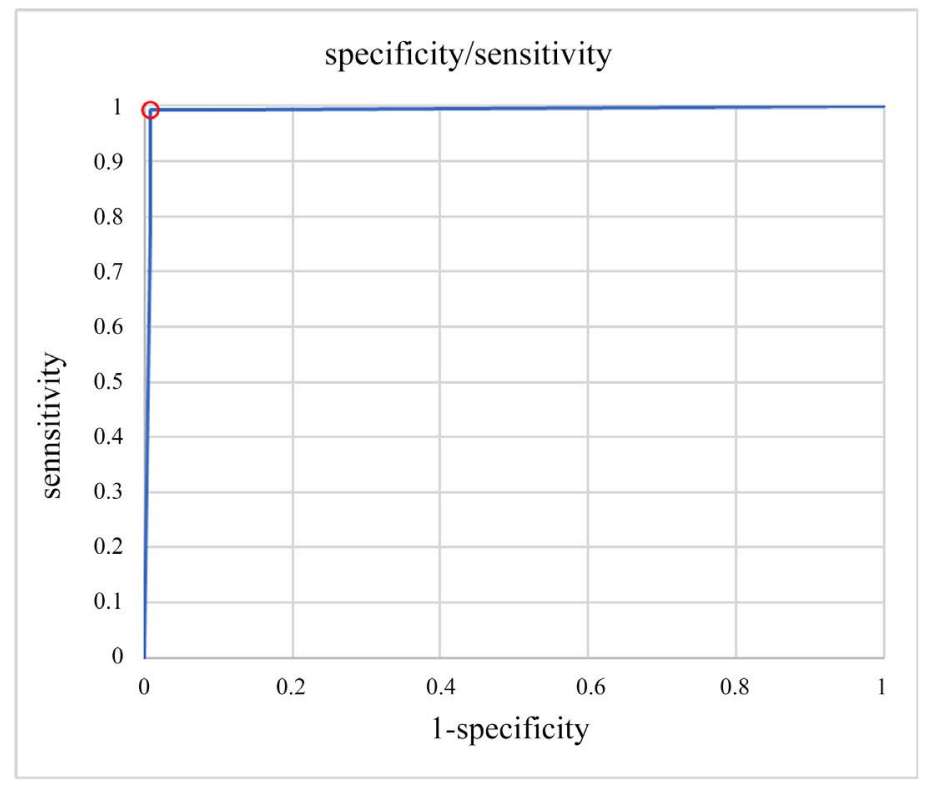

Figure 5. Specificity-sensitivity dependence in a sample of 536 measurements of COVID-19 diagnostics according to vibraimage parameters by measuring micromovements of the human head.

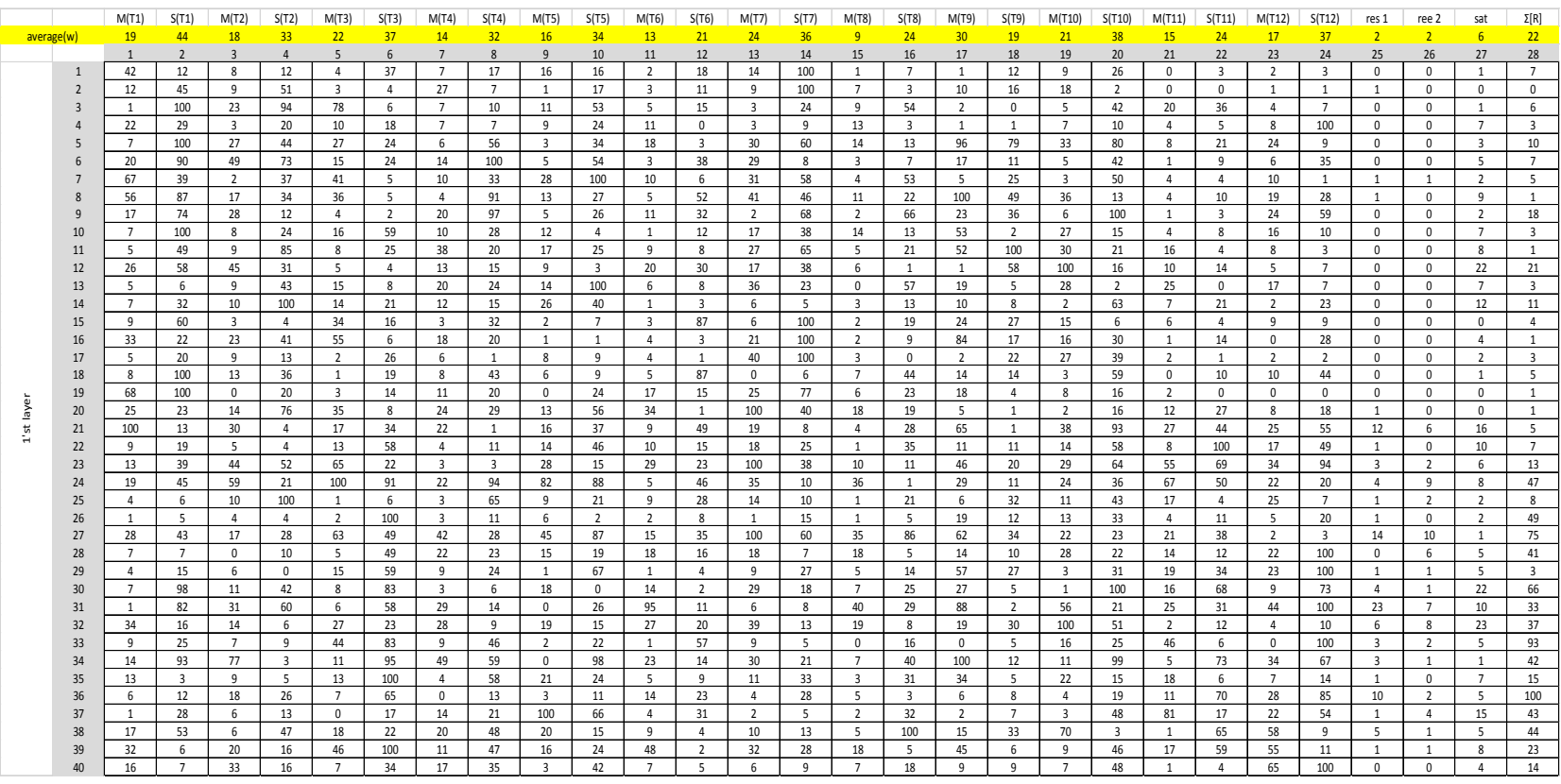

Figure 6. Average weights of input parameters on the first layer of feedforward neural network. 
Table 2. Detection of COVID-19 with normal integral health indicators by HealthTest program.

\begin{tabular}{cc}
\hline COVID-19 negative & COVID-19 positive \\
\hline Threshold & Threshold \\
0.5 & 0.5 \\
0.000005 & 0.999995 \\
0.000003 & 0.999999 \\
0.000002 & 0.999998 \\
0.000001 & 0.999999 \\
Probability & Probability \\
$99.25 \%$ & $99.25 \%$ \\
$95.52 \%$ & $96.27 \%$ \\
$91.42 \%$ & $93.66 \%$ \\
$90.30 \%$ & $92.16 \%$ \\
$89.18 \%$ & $91.42 \%$ \\
\hline
\end{tabular}

\section{Discussion}

The authors understand that presented statistics may change significantly with an increase in the number of measurements and is too early to draw conclusions about a confident $99 \%$ accuracy of the COVID-19 diagnostic by the method of head micromovements analyzing. Moreover, the number of having measurements significantly exceeds the number of studied patients (14 patients for 261 measurements). However, the above result allows us to look optimistically at the high accuracy of future COVID-19 diagnosis, since the discussed method has high reserves in providing additional information about the micromovements of the human head, primarily in an unlimited increase in the input parameters for AI processing. In addition, high sensitivity of vestibular-emotional reflex to detection of various diseases, including non-infectious ones, was previously shown and discussed [17]. An additional argument for the high accuracy of the proposed method for diagnosing COVID-19 is the ability to train AI on patients with different confirmation of COVID-19 (PCR, serology, CT), which allows avoiding systematic diagnostic errors inherent in each of these biochemical methods or radiology separately.

The ability to obtain the results of head micromovements at different stages of the disease is also a relative advantage of AI training in the proposed method. For example, it follows from Table 2 that the primary diagnostic program was unable to detect COVID-19 on certain days of the active phase of the disease (October 27 and 28) against the background of other symptoms of the disease. After adding these and other measurements of cases to the main database, the program successfully detects COVID-19 based on past results of measured behavioral parameters. Thus, almost every measurement of micromovements of a person head with confirmed COVID-19 provides unique information about the 
characteristics of the disease and can be used to train AI.

At the same time, the fundamental simplicity is great advantage of the proposed method, the studied person is tested only $1-3$ minutes in front of a standard web camera connected to a computer [16] [24]. Free AIvsCOVID software [25], opens unlimited possibilities for using this method for regular self-testing, pre-shift control, checks in places of public events, airports, concerts, train stations, etc.

A separate issue requires further study of the optimal time for measuring the micromovements of a human head for the most accurate diagnosis of COVID-19. In both groups of patients and the control group, the measurement time was 1 $(40 \%)$ and $3(60 \%)$ minutes for the same number of measurements. Presumably, the accuracy of determining the average median values of the parameters and their standard deviations slightly increases with 3-minute measurements [26], so now we recommend taking 3-minute measurements to diagnose COVID-19. However, we believe that in the course of further research it will be possible to reduce the testing time to 1 minute or even 30 seconds, due to an increase in the number of input controlled parameters when calculating the $\mathrm{AI}$ and the possible complication of the AI structure. Although according to the results of current measurements, all 4 errors ( 2 false positives and 2 false negatives) refer to 1 minute measurements, thus the accuracy of COVID-19 diagnostics for 3 minute measurements of head micromovements is $100 \%$.

The authors think that a slight decrease in the accuracy of the described method for diagnosing COVID-19 is possible with a significant increase in the number of measurements in the training samples. At present, with an insignificant amount of data in the samples, AI can artificially cling to vibraimage features inherent in cameras and measurement conditions, since the real vibraimage differs from the ideal vibraimage [24] [26]. With the increase in the amount of measurement data, these pseudo-features of COVID-19 will disappear, and whether this loss can be made up by trained $\mathrm{AI}$ is an open question that requires further study.

The active spread of COVID-19 in all countries requires a revision of traditional medical diagnostic methods and a more active development of non-contact computer methods, the representative of which is vibraimage technology combined with the capabilities of AI.

In this paper, we do not consider in details ANN structure, presented on the Figure 1 feedforward neural network was selected as enough simple, having low time for training and learning and having high reserves for improving. Also small having database and high-received accuracy are promising more optimization for the next study. Frankly speaking, we were surprised by the practical detection results received from so limited database on pretesting stage and in our understanding it confirms high potential accuracy of suggested method and current AIvsCOVID [25] program trained on a doubled database.

The authors express their readiness to cooperate with various teams to next 
improvements of developed method, increase the database for AI training, and improve the accuracy and reliability of the results obtained.

\section{Conclusions}

We have developed and offered free COVID-19 test program [25] with an estimated accuracy better than $99 \%$. In our opinion, its active implementation in all areas will be able to stop the pandemic, and joint efforts to refine it will be able to make it more reliable and possibly universal for detecting new yet unknown diseases. Only regular selftesting and testing all people contact you, will help to stop COVID-19 pandemic.

A global pandemic requires a world effort to end it-none of us will be safe until everyone is safe. Access to vaccines, tests and treatments for everyone who needs them is the only way out-this is a historic test for global cooperation [27]. Testing time and accuracy are so important as vaccines and treatments.

\section{Ethics Approval and Consent to Participate}

Authors do not see ethics problems in the current study.

Ethical and professional principles were followed throughout the study. All participants provided written informed consent prior to study entry. All participants could discontinue the prescribed interventions (research) of their choice.

\section{Conflicts of Interest}

The authors declare no conflicts of interest regarding the publication of this paper.

\section{References}

[1] WHO (2020) Weekly Operational Update on COVID-19. https://www.who.int/publications/m/item/weekly-operational-update---30-october$\underline{2020}$

[2] Wynants, L., et al. (2020) Prediction Models for Diagnosis and Prognosis of Covid-19: Systematic Review and Critical Appraisal. BMJ, 369, m1328.

[3] Soares, et al. (2020) A Novel High Specificity COVID-19 Screening Method Based on Simple Blood Exams and Artificial Intelligence.

[4] Ching, L., et al. (2020) COVID-19 Special Column: Principles behind the Technology for Detecting SARS-CoV-2, the Cause of COVID-19. Hawai $i$ Journal of Health \& Social Welfare, 79, 136-142.

[5] Kopel, J., et al. (2020) Antibody Tests for COVID-19. Baylor University Medical Center Proceedings, 34, 63-72. https://doi.org/10.1080/08998280.2020.1829261

[6] Sheikhzadeh, E., et al. (2020) Diagnostic Techniques for COVID-19 and New Developments. Talanta, 220, Article ID: 121392. https://doi.org/10.1016/j.talanta.2020.121392

[7] Bastos, M.L., et al. (2020) Diagnostic Accuracy of Serological Tests for Covid-19: Systematic Review and Meta-Analysis. BMJ, 370, m2516. https://doi.org/10.1136/bmj.m2516

[8] Kamkin, E.G. (2020) Interim Methodological Recommendations Prevention, Diagnostics and Treatment of New Coronavirus Infection (COVID-19). Ministry of Health 
of the Russian Federation, Version 8. (In Russian)

https://static-0.minzdrav.gov.ru/system/attachments/attaches/000/051/777/original/ 030902020 COVID-19 v8.pdf

[9] Laguarta, J., et al. (2020) COVID-19 Artificial Intelligence Diagnosis Using Only Cough Recordings. IEEE Open Journal of Engineering in Medicine and Biology.

https://ieeexplore.iee.org/stamp/stamp.jsp?tp=\&arnumber=9208795 https://doi.org/10.1109/OJEMB.2020.3026928

[10] Bwire, G.M. and Paulo, L.S. (2020) Coronavirus Disease-2019: Is Fever an Adequate Screening for the Returning Travelers? Tropical Medicine and Health, 48, 14. https://doi.org/10.1186/s41182-020-00201-2

[11] Jendrny, P., et al. (2020) Scent Dog Identification of Samples from COVID-19 Patients: A Pilot Study. BMC Infectious Diseases, 20, Article No. 536.

https://doi.org/10.1186/s12879-020-05281-3

[12] Report (2020) Report of the WHO-China Joint Mission on Coronavirus Disease 2019 (COVID-19).

https://www.who.int/docs/default-source/coronaviruse/who-china-joint-mission-o n-covid-19-final-report.pdf

[13] Minkin, V. and Nikolaenko, Y. (2017) Application of New Concept for Multiple Intelligences Calculation for Personality and Social Groups Comparison Research. Journal of Behavioral and Brain Science, 7, 447-463.

https://doi.org/10.4236/jbbs.2017.710032

[14] Minkin, V. and Myasnikova, E. (2018) Using Vibraimage Technology to Analyze the Psychophysiological State of a Person during Opposite Stimuli Presentation. Journal of Behavioral and Brain Science, 8, 218-239.

[15] Minkin, V.A. and Bobrov, A.F. (2020) Health Diagnostics Using Assessment of Physiological Systems Signals Desynchronization. First Results of HealthTest Program Practical Applications. Proceedings of the 3rd International Open Science Conference, St. Petersburg, 25-26 June 2020, 292-300.

[16] Minkin, V. (2020) Vibraimage, Cybernetics and Emotions. Renome, St. Petersburg.

[17] Blank, M.A., et al. (2014) Method for Screening Prostate Cancer Diagnosis. RU Patent No. 2515149

[18] Minkin, V.A. and Nikolaenko, N.N. (2008) Application of Vibraimage Technology and System or Analysis of Motor Activity and Study of Functional State of the Human Body. Biomedical Engineering, 42, 196-200. https://doi.org/10.1007/s10527-008-9045-9

[19] Haykin, S. (2009) Neural Networks and Learning Machines. Third Edition, Pearson Education, Inc., McMaster University, Hamilton. http://dai.fmph.uniba.sk/courses/NN/haykin.neural-networks.3ed.2009.pdf

[20] VibraHT (2020) Health Test Professional Program. Manual. http://www.psymaker.com/downloads/VibraHTEng.pdf

[21] HealthTest (2020) Health Testing Program. Manual. http://www.psymaker.com/downloads/HealthTestEng.pdf

[22] Huang, et al. (2020) Timely Diagnosis and Treatment Shortens the Time to Resolution of Coronavirus Disease (COVID-19) Pneumonia and Lowers the Highest and Last CT Scores from Sequential Chest CT. American Journal of Roentgenology, 215, 367-373. https://doi.org/10.2214/AJR.20.23078

[23] 258-FZ (2020) On Experimental Legal Regimes in the Sphere of Digital Innovation in the Russian Federation. 
http://publication.pravo.gov.ru/Document/View/0001202007310024

[24] Minkin, V.A. (2017) Vibraimage. Renome, St. Petersburg.

[25] AIvsCOVID (2020) Covid-19 Diagnoses Program. Manual. http://www.psymaker.com/downloads/AIvsCOVID.pdf

[26] Minkin, V.A. (2019) About the Accuracy of Vibraimage Technology. The 2nd International Open Science Conference Modern Psychology, St. Petersburg, 25-26 June, 2019, 294-305

[27] Ghebreyesus, T.A. and von der Leyen, U. (2020) A Global Pandemic Requires a World Effort to End It-None of Us Will Be Safe until Everyone Is Safe https://www.who.int/news-room/commentaries/detail/a-global-pandemic-requiresa-world-effort-to-end-it-none-of-us-will-be-safe-until-everyone-is-safe 